\title{
A SECOND ORDER NONLINEAR OSCILLATION THEOREM ${ }^{1}$
}

\author{
JAMES S. W. WONG
}

\begin{abstract}
An oscillation criterion is given for the second order nonlinear equation $x^{\prime \prime}+a(t)|x|^{\gamma} \operatorname{sgn} x=0, \gamma>1$, where the coefficient $a(t)$ is not assumed to be nonnegative for all large values of $t$.
\end{abstract}

Consider the second order nonlinear differential equation

$$
x^{\prime \prime}+a(t)|x|^{\gamma} \operatorname{sgn} x=0, \quad \gamma>0,
$$

where $a(t) \in C[0, \infty)$. We restrict our attention to solutions of (1) which exist on some ray $\left[t_{0}, \infty\right)$, where $t_{0} \geqq 0$ may depend on the particular solution. Such a solution is said to be oscillatory if it has arbitrarily large zeros. Equation (1) is called oscillatory if all such solutions are oscillatory. For a general discussion on nonlinear oscillation problems, we refer the reader to [14]. We are here concerned with sufficient conditions on $a(t)$ for the oscillation of (1) when $a(t)$ is allowed to assume negative values for arbitrarily large values of $t$. The well-known Wintner-Leighton oscillation criterion for the linear equation, i.e., equation (1) when $\gamma=1$, states that if $a(t)$ satisfies

$$
\lim _{T \rightarrow \infty} \int_{0}^{T} a(t) d t=+\infty,
$$

then equation (1) is oscillatory for $\gamma=1$, see [12], [8]. Waltman [11] showed that condition (2) is also sufficient for the oscillation of (1) when $\gamma>1$. Bhatia [1] and Wong [13] independently generalized Waltman's result to more general nonlinear equations showing in particular that condition (2) is sufficient for oscillation of (1) for all $\gamma>0$.

We note that Wintner [12] in fact proved a stronger result, namely, if $a(t)$ satisfies the weaker condition

$$
\lim _{T \rightarrow \infty} \frac{1}{T} \int_{0}^{T} \int_{0}^{t} a(s) d s d t=+\infty,
$$

Received by the editors December 27, 1972.

AMS (MOS) subject classifications (1970). Primary 34C10, 34C15.

Key words and phrases. Second order equations, nonlinear, oscillation.

1 Research supported in part by Army Research Office, Durham, through Contract No. DA-ARO-D31-24-72-G95. 
then equation (1) is oscillatory for $\gamma=1$. Clearly, condition (2) implies condition (3). It is therefore of interest to investigate whether the weaker hypothesis (3) is sufficient for the oscillation of (1) for all $\gamma>0$. Recently, Kamenev [6] proved that for $0<\gamma<1$, the weaker condition

$$
\limsup _{T \rightarrow \infty} \frac{1}{T} \int_{0}^{T} \int_{0}^{t} a(s) d s d t=+\infty,
$$

suffices for the oscillation of (1). The purpose of this note is to provide a partial solution to this problem by proving that condition (4) together with the following condition

$$
\liminf _{t \rightarrow \infty} \int_{0}^{t} a(s) d s=-\lambda>-\infty, \quad \lambda>0,
$$

imply oscillation. This gives credence to the conjecture that the original Wintner's oscillation criterion (4) remains valid for equation (1). Clearly, condition (2) implies condition (5), hence our result generalizes that of Waltman.

We now state and prove the following

THEOREM. Suppose that a(t) satisfies conditions (4) and (5), then equation (1) is oscillatory when $\gamma>1$.

Proof. Assume the contrary, then there exists a solution $x(t)$ which may be assumed to be positive on $\left[t_{0}, \infty\right)$ for some $t_{0} \geqq 0$. Dividing (1) through by $x^{\gamma}(t)$ and integrating from $t_{0}$ to $t$, we obtain

$$
x^{-\gamma}(t) x^{\prime}(t)+\gamma \int_{t_{0}}^{t}\left(\frac{x^{\prime}(s)}{x^{\beta}(s)}\right)^{2} d s+A(t)=c_{1},
$$

where $\beta=(\gamma+1) / 2, \quad c_{1}=x^{-\gamma}\left(t_{0}\right) x^{\prime}\left(t_{0}\right)$, and $A(t)$ denotes the indefinite integral $\int_{t_{0}}^{t} a(s) d s$. Integrating (6) once more from $t_{0}$ to $t$, we obtain

$$
\frac{1}{1-\gamma} x^{-\gamma+1}(t)+\gamma \int_{t_{0}}^{t} \int_{t_{0}}^{s}\left(\frac{x^{\prime}(\tau)}{x^{\beta}(\tau)}\right)^{2} d \tau d s+\int_{t_{0}}^{t} A(s) d s=c_{1} t+c_{2}
$$

where $c_{2}=1 /(1-\gamma) x^{-\gamma+1}\left(t_{0}\right)$.

We distinguish three cases of the behaviour of $x^{\prime}(t)$, namely, (i) $x^{\prime}(t)$ oscillatory on $\left[t_{0}, \infty\right)$, (ii) $x^{\prime}(t)>0$ on $\left[t_{*}, \infty\right)$ for some $t_{*} \geqq t_{0}$, and (iii) $x^{\prime}(t)<0$ on $\left[t_{*}, \infty\right)$ for some $t_{*} \geqq t_{0}$, and show that the assumption $x(t)>0$ leads to contradiction in each case.

Suppose that $x^{\prime}(t)$ is oscillatory, then there exists a sequence $\left\{t_{n}: n=1,2\right.$, $3, \cdots\}$ such that $x^{\prime}\left(t_{n}\right)=0$ and $t_{n} \rightarrow \infty$. It follows from (6) and condition 
(5) that $x^{\prime} x^{-\beta}(t) \in L^{2}\left(t_{0}, \infty\right)$. By Schwarz's inequality, we note

$$
\frac{1}{(1-\beta)^{2}} \frac{1}{x^{2 \beta-2}(t)} \leqq\left|\int_{t_{0}}^{t} \frac{x^{\prime}(s)}{x^{\beta}(s)} d s\right|^{2} \leqq t \int_{t_{0}}^{t}\left(\frac{x^{\prime}(s)}{x^{\beta}(s)}\right)^{2} d s .
$$

Recall that $2 \beta-2=\gamma-1$, and so (8) implies that there exists a constant $M$ such that

$$
x^{-\gamma+1}(t) /(\gamma-1) \leqq M t, \quad t \geqq t_{0} .
$$

Applying the estimate (9) in (7), we obtain the following inequality, valid for all $t \geqq t_{0}$,

$$
-M t+\int_{t_{0}}^{t} A(s) d s \leqq c_{1} t+c_{2},
$$

which contradicts condition (4).

Next, suppose that $x^{\prime}(t)>0$ for $t \geqq t_{1} \geqq t_{0}$, thus $x(t) \geqq x\left(t_{0}\right)$ and $x^{-\gamma+1}(t) \leqq$ $x^{-\gamma+1}\left(t_{0}\right)$. We deduce from (7) that

$$
\frac{1}{1-\gamma} x^{-\gamma+1}\left(t_{0}\right)+\int_{t_{0}}^{t} A(s) d s \leqq c_{1} t+c_{2}
$$

which again contradicts condition (4).

Finally, we assume that $x^{\prime}(t)<0$ for $t \geqq t_{*} \geqq t_{0}$. By condition (5), we can estimate (6) as follows,

$$
-\frac{x^{\prime}(t)}{x^{\gamma}(t)} \geqq-\left(c_{1}+\lambda\right)+\gamma \int_{t_{0}}^{t} \frac{x^{\prime 2}(s)}{x^{\gamma+1}(s)} d s .
$$

If the integral in (10) is finite as $t \rightarrow \infty$, we can deduce a contradiction in a similar way as the case when $x^{\prime}(t)$ is oscillatory. Otherwise we may choose $T \geqq t_{*}$ such that

$$
\gamma \int_{t_{0}}^{T} \frac{x^{\prime 2}(s)}{x^{\gamma+1}(s)} d s=1+c_{1}+\lambda
$$

For $t \geqq T$, we multiply (10) through by

$$
-\frac{x^{\prime}(t)}{x(t)}\left\{-\left(c_{1}+\lambda\right)+\gamma \int_{t_{0}}^{t} \frac{x^{\prime 2}(s)}{x^{\gamma+1}(s)} d s\right\}
$$

and integrate from $T$ to $t$ to obtain

$$
\log \left(-\left(c_{1}+\lambda\right)+\gamma \int_{t_{0}}^{t} \frac{x^{\prime 2}(s)}{x^{\gamma+1}(s)} d s\right) \geqq \gamma \int_{T}^{t} \frac{-x^{\prime}(s)}{x(s)} d s,
$$

which together with (10) yields

$$
-x^{\prime}(t) / x^{\gamma}(t) \geqq x^{\gamma}(T) / x^{\gamma}(t) .
$$


It follows from (11) that $x^{\prime}(t) \leqq-x^{\gamma}(T)<0$, which contradicts the assumption that $x(t)>0$. This completes the proof.

Remark 1. Conditions (4) and (5) are also sufficient for the oscillation of (1) with $\gamma=1$. The validity of this result for the linear case follows from a result of Hartman [5], see also Coles [3] and Macki and Wong [9].

REMARK 2. For $0<\gamma<1$, condition (4) gives an immediate contradiction in (7). This offers an alternate argument for Kamenev's result.

REMARK 3. Erbe [4] gives another interesting generalization of Waltman's result. Erbe introduced the following condition on $a(t)$ that for all large $t$

$$
\liminf _{t \rightarrow \infty} \int_{T}^{t} a(s) d s>0,
$$

and showed that if $a(t)$ satisfies (12) and that the linear equation

$$
u^{\prime \prime}+\lambda a(t) u=0
$$

is oscillatory for all $\lambda>0$, then equation (1) is oscillatory. We note that although condition (3) or conditions (4) and (5) are sufficient for the oscillation of (13) for all $\lambda>0$, condition (12) is not implied by condition (5), (in fact, the converse is true). This shows that our result is not compatible with that of Erbe's. Condition (12) was also used in Bobisud [2], see also Onose [10].

Finally, we present an example of $a(t)$ which satisfies conditions (4) and (5) but fails to satisfy (12). Thus, Erbe's result does not apply in this case. Define

$$
\begin{aligned}
a(t) & =\alpha_{k} t+\beta_{k}, & & 4 k \leqq t \leqq 4 k+1 \\
& =\gamma_{k} t+\delta_{k}, & & 4 k+1 \leqq t \leqq 4 k+2 \\
& =\xi_{k} t+\eta_{k}, & & 4 k+2 \leqq t \leqq 4 k+3 \\
& =\sigma_{k} t+\tau_{k}, & & 4 k+3 \leqq t \leqq 4 k+4,
\end{aligned}
$$

where $\alpha_{k}=(k+1)+\sum_{i=1}^{k}\left(1 / 2^{i}\right), \beta_{k}=-(4 k) \alpha_{k} ; \gamma_{k}=-\alpha_{k}, \delta_{k}=-(4 k+2) \gamma_{k}$; $\xi_{k}=-\alpha_{k}-\left(1 / 2^{k+1}\right), \quad \eta_{k}=-(4 k+2) \xi_{k} ; \quad \sigma_{k}=-\xi_{k}, \tau_{k}=-(4 k+4) \sigma_{k}$. It is easy to see that $a(t) \in C[0, \infty)$ and satisfies

$$
\liminf _{t \rightarrow \infty} a(t)=-\infty, \quad \limsup _{t \rightarrow \infty} a(t)=+\infty .
$$

Denote $A(t)=\int_{0}^{t} a(s) d s$. It is readily verified that

$$
\begin{aligned}
A(4 k) & =-\sum_{i=1}^{k} \frac{1}{2^{i}}, & A(4 k+1) & =\frac{k+1}{2}-\frac{1}{2} \sum_{i=1}^{k} \frac{1}{2^{i}}, \\
A(4 k+2) & =k+1, & A(4 k+3) & =\frac{k+1}{2}-\frac{1}{2} \sum_{i=1}^{k+1} \frac{1}{2^{i}} .
\end{aligned}
$$


From this we deduce $\liminf _{t \rightarrow \infty} A(t)=-1, \quad \lim \sup _{t \rightarrow \infty} A(t)=+\infty$. Denote $\mathscr{A}(T)=(1 / T) \int_{0}^{T} A(t) d t$. We compute and find

$$
\begin{aligned}
& \mathscr{A}(4 n+4)= \frac{1}{4(n+1)} \sum_{k=0}^{n}[A(4 k)+A(4 k+1) \\
&\quad+A(4 k+2)+A(4 k+3)] \\
&= \frac{1}{4(n+1)} \sum_{k=0}^{n}\left(2(k+1)-2 \sum_{i=1}^{k} \frac{1}{2^{i}}-\frac{1}{2^{k+1}}\right) \\
& \geqq \frac{1}{4(n+1)} \sum_{k=1}^{n} 2 k \\
&=\frac{1}{4(n+1)} n(n+1)=\frac{n}{4},
\end{aligned}
$$

which shows that $a(t)$ satisfies condition (4).

\section{REFERENCES}

1. N. P. Bhatia, Some oscillation theorems for second order differential equations, J. Math. Anal. Appl. 15 (1966), 442-446. MR 34 \#3017.

2. L. E. Bobisud, Oscillation of nonlinear second order equations, Proc. Amer. Math. Soc. 23 (1969), 501-505. MR 40 \#448.

3. W. J. Coles, An oscillation criterion for second order linear differential equations, Proc. Amer. Math. Soc. 19 (1968), 755-759.

4. L. Erbe, Oscillation theorems for second order nonlinear differential equations, Proc. Amer. Math. Soc. 24 (1970), 811-814. MR 40 \#5973.

5. P. Hartman, On non-oscillatory linear differential equations of second order, Amer. J. Math. 74 (1952), 389-400. MR 14, 50.

6. I. V. Kamenev, Certain specifically nonlinear oscillation theorems, Mat. Zametki 10 (1971), 129-134. (Russian) MR 44 \#4284.

7. I. T. Kiguradze, $A$ note on the oscillation of solutions of the equation $u^{\prime \prime}+$ $a(t)|u|^{n}$ sgn $u=0$, Casopis Pèst. Mat. 92 (1967), 343-350. (Russian) MR 36 \#4064.

8. W. Leighton, The detection of the oscillation of solutions of a second order linear differential equation, Duke Math. J. 17 (1950), 57-61. MR 11, 248, 871.

9. J. W. Macki and J. S. W. Wong, Oscillation theorems for linear second order ordinary differential equations, Proc. Amer. Math. Soc. 20 (1969), 67-72. MR 38 \#3513.

10. H. Onose, On oscillation of nonlinear second order equations, J. Math. Anal. Appl. 39 (1972), 122-124.

11. P. Waltman, An oscillation criterion for a nonlinear second order equation, J. Math. Anal. Appl. 10 (1965), 439-441. MR 30 \#3265.

12. A. Wintner, A criterion of oscillatory stability, Quart. Appl. Math. 7 (1949), 115-117. MR 10, 456.

13. J. S. W. Wong, On two theorems of Waltman, SIAM J. Appl. Math. 14 (1966), 724-728. MR 34 \#6228.

14. - On second order nonlinear oscillation, Funkcial. Ekvac. 11 (1969), 207-234. MR 39 \#7221.

Department of Mathematics, University of Iowa, Iowa City, Iowa 52240 